\title{
Leisure activities are linked to mental health benefits by providing time structure: comparing employed, unemployed and homemakers
}

\author{
William K Goodman, Ashley M Geiger, Jutta M Wolf
}

\begin{abstract}
- Additional material is published online only. To view please visit the journal online (http://dx.doi.org/10.1136/jech2016-207260).

Psychology Department, Brandeis University, Waltham, Massachusetts, USA

\section{Correspondence to} Dr Jutta M Wolf, Psychology Department, Brandeis University, 415 South St. MS062, Waltham, MA 02454, USA; jmw@brandeis.edu
\end{abstract}

Received 2 February 2016 Revised 19 April 2016 Accepted 23 May 2016 Published Online First 13 June 2016

\section{CrossMark}

\footnotetext{
To cite: Goodman WK, Geiger AM, Wolf JM. J Epidemiol Community Health 2017:71:4-11.
}

\begin{abstract}
Background Unemployment has consistently been linked to negative mental health outcomes, emphasising the need to characterise the underlying mechanisms. The current study aimed at testing whether compared with other employment groups, fewer leisure activities observed in unemployment may contribute to elevated risk for negative mental health via loss of time structure. Methods Depressive symptoms (Center for Epidemiologic Studies Depression), leisure activities (exercise, self-focused, social), and time structure (Time Structure Questionnaire (TSQ)) were assessed cross-sectionally in 406 participants (unemployed=155, employed $=140$, homemakers $=111$ ) recruited through Amazon Mechanical Turk.

Results Controlling for gender and age, structural equation modelling revealed time structure partially (employed, homemakers) and fully (unemployed) mediated the relationship between leisure activities and depressive symptoms. With the exception of differential effects for structured routines, all other TSQ factors (sense of purpose, present orientation, effective organisation and persistence) contributed significantly to all models.

Conclusions These findings support the idea that especially for the unemployed, leisure activities impose their mental health benefits through increasing individuals' perception of spending their time effectively. Social leisure activities that provide a sense of daily structure may thereby be a particularly promising low-cost intervention to improve mental health in this population.
\end{abstract}

\section{INTRODUCTION}

Unemployment has consistently been linked to negative mental health outcomes, ${ }^{1}$ including elevated risk for depression. ${ }^{2-4}$ This emphasises the need to characterise the mechanisms underlying how unemployment negatively influences mental health. Two factors and their interplay will be examined in the current study: recreational activities and time structure. Previous studies have indicated significant detrimental effects of unemployment on physical activity, ${ }^{5}$ leisure activities $^{6}$ and social contacts/interactions. Interestingly, each of these activities provides time structure to various degrees. Further, employment by nature imposes a structured routine on an individual, ${ }^{8}$ defines the passage of time through regular activity, and provides a sense of purpose. Not surprisingly, previous research suggests that unemployed individuals have reduced time structure, ${ }^{9}$ and this, in turn, is linked to poorer mental health ${ }^{10}$ and overall well-being. ${ }^{11}$ Hence, the current study aimed at testing whether dependent on employment status, fewer leisure activities may contribute to higher risk for negative mental health via decreased time structure.

Physical activity has been shown to have numerous psychological benefits ${ }^{12-17}$ and has been effectively used as an intervention for depression. ${ }^{18}$ Similarly, recreational activities contribute to better moods and reduced depressive symptoms. ${ }^{19} \quad 20$ However, different types of recreational leisure activities may not confer equal benefits for depressive symptoms. Recent research suggests that social leisure activities may have stronger associations with depressive symptoms than exercise. ${ }^{21}$ While still beneficial, links between depressive symptoms and self-focused leisure activities, such as watching TV, were weaker in the unemployed. These findings raise the question of why employed and unemployed individuals differ in the extent to which social versus self-focused leisure activities can improve mental health. One explanation may be that those activities differ in terms of how much time structure they provide.

Time structure assesses how effectively individuals perceive they spend their time with higher levels of time structure associated with better mental and physical health outcomes. ${ }^{10}{ }^{22}$ However, time structure was originally conceptualised and defined as a multidimensional concept composed of sense of purpose, structured routine, present orientation, effective organisation and persistence. ${ }^{23}$ With the exception of a small number of other studies, ${ }^{23-28}$ most research has not examined the individual contributions of each subscale to the latent concept of time structure, despite the multiple facets being only weakly correlated. ${ }^{29}$ To date, it is thus not clear whether only specific combinations of time structure facets are linked to negative mental health outcomes and, if so, whether this pattern may differ in unemployed individuals.

A small number of studies have assessed the role of leisure activities in providing time structure, and findings suggest that leisure activities specifically provide sense of purpose as well as meaning. ${ }^{6} 30$ Similarly, leisure activities were found to serve as distractions from everyday worries and to provide daily structure, ${ }^{31}$ while exercise, in particular, was noted to increase structured routines. ${ }^{31}$ Together, these findings suggest that leisure activities influence how an individual perceives time structure. However, no study tested how various types of leisure activities (social, self-focused, exercise) contribute differently to perceptions of time structure, 
and how this may differ in employed and unemployed individuals. Furthermore, it is not known to what extent specific combinations of time structure factors mediate these links between leisure activities and depressive symptoms.

While unemployment presents a special situation in terms of links between leisure activities, time structure and mental health, the same is true for another group of 'out of the labor force' (OLF) individuals. Although infrequently studied, decreases in time structure along with decreases in mental health have been reported among the general OLF population. ${ }^{10}$ OLF individuals include students and retirees, however, in the current context, homemakers are of specific relevance. On one hand, homemakers do not have an income, and thus, can be regarded as unemployed. At the same time, however, they often are involved in activities imposing time structure, such as caring for children. ${ }^{32}$ Therefore, homemakers are generally required to generate an internally imposed sense of time structure in contrast with their employed counterparts who have time structure externally imposed due to employment responsibilities. As such, assessing homemakers is a promising approach to further elucidate the relationships between leisure activities, depressive symptoms and internally versus externally imposed time structure.

In summary, the current study aimed at determining how the relationships between leisure (social, self-focused and exercise) activities and depressive symptoms are influenced by time structure, and whether these relationships differ for employed and unemployed individuals. The second aim was to examine the contributions of the various facets of time structure towards the overall measure of time structure in both employment groups. Finally, we aimed at assessing the above relationships in the understudied occupation of homemaking.

\section{METHODS}

\section{Participants}

Participants were 567 US residents who were 18 years old or older, and had earned an Amazon's Mechanical Turk Human Intelligence Tasks rate of $98 \%$ or better. Recruitment was controlled for employment status, and participants were excluded for spending $<15 \mathrm{~min}$ on the survey, or providing two or more straight-lined responses $(n=68)$.

Only participants who self-reported to be employed full time $(n=140)$, unemployed $(n=155)$ or 'homemaker' $(n=111)$ were included. Excluded participants were the retired $(n=16)$, parttime workers $(n=47)$ and male homemakers $(n=9)$.
Furthermore, 21 participants were excluded due to not providing answers to entire questionnaire(s), resulting in a final study population of $n=406$ (age: $33.9 \pm 10.2$ years; see table 1 ). The study was approved by the local IRB, and participants were compensated US $\$ 2$ per hour.

\section{Procedures}

Participants were recruited (in 2012) through Amazon's Mechanical Turk (mTurk), an online marketplace where registered users complete tasks for payment. It has been found that mTurk was more demographically diverse than traditional sampling methods, ${ }^{33}$ such as university campuses, and accurately represents the US population. ${ }^{34}$ Interested users saw a description of the study and gave informed consent via electronic acceptance of study materials. They were then redirected to Qualtrics (http://www.qualtrics.com), the platform used to administer the survey which included the measures described below as part of a study previously described. ${ }^{21}$

\section{Measures}

Center for Epidemiologic Studies Depression (CESD) scale: The CESD scale is a 20 -item measure assessing frequency of depressive symptoms over the last month. ${ }^{35}$ Responses range from 0 (rarely) to 3 (most or all of the time) with higher scores indicating more depressive symptoms. Scores of 16 or higher are indicative of potentially clinically relevant symptom severity.

Pittsburgh Enjoyable Activities Test (PEAT): The 10-item PEAT examines participation in leisure activities $(\alpha=0.72) .{ }^{30}$ Responses range from 0 (never) to 4 (everyday). In line with earlier research, ${ }^{21}$ items (1) 'Spending quiet time alone'; (2) 'Spending time unwinding'; (7) 'Vacationing'; (8) 'Communing with nature' and (10) 'Hobbies' were summed to create a selffocused recreational activities score. Items (3) 'Visiting others'; (4) 'Eating with others'; (5) 'Doing fun things with others' and (6) 'Club, fellowship and religious group participation' were summed to reflect social recreational activities. Item (9) assessed 'Sports and Exercise'.

Time Structure Questionnaire (TSQ): The TSQ is a 26-item questionnaire examining how effectively a person perceives they use their time. ${ }^{23}$ It is composed of items like 'Do you plan your activities from day to day?' and 'Do you ever have trouble organizing the things you have to do?'. Responses range from 1 (yes, always) to 7 (no, never), with higher scores indicating more time structure.

Table 1 Participant demographics (all parenthetical values represent SD unless otherwise noted)

\begin{tabular}{lcccc}
\hline & Employed & Unemployed & Homemakers & Overall \\
\hline Age & $35.03(9.70)$ & $33.37(11.53)$ & $33.40(8.90)$ & $0(112)$ \\
Gender (females) & $62(78)$ & $85(70)$ & $17.27(13.16)$ & $147(260)$ \\
CESD & $15.91(12.24)$ & $22.39(12.97)$ & $2.35(1.01)$ & $18.76(13.07)$ \\
Self-focused recreation & $2.31(0.63)$ & $2.3(0.70)$ & $2.61(0.99)^{* *}$ & $2.32(0.78)$ \\
Social recreation & $2.28(0.88)$ & $2.05(0.89)$ & $2.30(1.55)$ & $2.28(0.94)$ \\
Exercise & $2.41(1.53)$ & $2.10(1.55)$ & $111.03(21.53)$ & $2.26(1.55)$ \\
Time structure & $111.26(20.19)$ & $97.88(17.26)^{* *}$ & $22.37(6.73)$ & $106.10(20.52)$ \\
Sense of purpose & $21.7(6.89)$ & $18.24(6.51)^{* * *}$ & $21.31(3.68)$ & $20.57(6.94)$ \\
Structured routine & $20.85(3.67)$ & $20.35(3.65)$ & $10.57(3.57)$ & $20.78(3.68)$ \\
Present orientation & $10.07(3.97)$ & $9.39(3.67)^{*}$ & $17.55(5.99)$ & $1.95(3.77)$ \\
Effective organisation & $18.94(6.17)$ & $14.77(5.25)^{* * *}$ & $15.50(4.00)$ & $16.97(6.05)$ \\
Persistence & $15.92(3.98)$ & $14.14(3.88)^{* *}$ & &
\end{tabular}




\section{Statistical analysis}

The initial proposed model consisted of the latent variable ('Time structure') loading onto all subscales of the TSQ and controlled for age and gender. Next, the latent construct, along with one of the specific recreational activities, and employment status, was used to predict depressive symptoms (figures 1-3). In more detail, a structural model was constructed to examine loadings of each TSQ subscale and the latent variable encompassing time structure. For each employment group, a structural equation model was constructed to evaluate the direct effects between the latent variable (time structure) and a specific activity (social, self-focused, exercise) and depressive symptoms, as well as the potential mediating effects of time structure. Mediational analysis was conducted using IBM AMOS (22) bootstrap analysis for indirect effects using 2000 bootstrap iterations and percentile-based levels of significance. Following standard guidelines, ${ }^{36}$ model fits were determined using the root mean square error of approximation (RMSEA). The criterion of 0.1 was used to delineate between good and poor-fitting models. Statistical tests not otherwise controlling for multiple comparisons were interpreted using Bonferroni-adjusted levels of a priori significance.

\section{RESULTS}

\section{Preliminary analyses}

Employed and unemployed did not differ in terms of gender distribution $\left(\chi^{2}(1)=3.28, p=0.07\right)$, and as pointed out earlier, homemakers were exclusively female. No differences were observed in self-reported depressive symptoms between the employed and homemakers, while the unemployed reported significantly elevated depressive symptoms $(\mathrm{F}(4401)=6.87, \mathrm{p}<0.001)$. Similarly, exercise frequency $\left(\chi^{2}(8)=7.72, p=0.46\right)$ and self-focused leisure $(\mathrm{F}(2401)=0.61, \mathrm{p}=0.66)$ did not differ between the three groups, with the exception that homemakers engaged in social leisure activity more frequently $(\mathrm{F}(2401)=6.17, \mathrm{p}<0.001)$. In terms of time structure, the employed, unemployed, and homemakers reported comparable usage of structured routines $(\mathrm{F}(2401)=1.38$, $\mathrm{p}=0.24)$. However, significant differences were observed between the three groups for all other facets of time structure: sense of purpose $(\mathrm{F}(2401)=9.15, \mathrm{p}<0.001)$, effective organisation $(\mathrm{F}$ $(2401)=13.37, \mathrm{p}<0.001)$, persistence $(\mathrm{F}(2401)=6.15, \mathrm{p}=0.001)$ and present orientation $(\mathrm{F}(2401)=12.36, \mathrm{p}<0.001$; difference between homemakers and the unemployed only). The pattern was consistent across the four facets, such that the unemployed selfreported lower levels of time structure than both the employed and homemakers, while no differences were observed between the employed and homemakers. Assessing the overall construct of time structure, unemployed participants $(\mathrm{M}=97.88 \pm 17.26)$ reported a lower sense of time structure than employed individuals $(111.26 \pm 20.19)$ and homemakers (111.03 $\pm 21.53 ; \mathrm{F}(2401)$ $=17.11, \mathrm{p}<0.001)$.

Next, we examined the simple relationships between leisure activity and TSQ subscales controlling for age and gender (see table 2). Applying Bonferroni correction, two main patterns were revealed. Specifically, sense of purpose was related to all recreational activities for employed participants (all $\mathrm{p}<0.001$ ), to social and self-focused recreation for unemployed (all $\mathrm{p}<0.001$ ), and to socially based leisure for homemakers $(p<0.001)$. Contrarily, effective organisation was related to all recreational activities for unemployed individuals (all $\mathrm{p}<0.001$ ), while no relationships were observed between recreation and effective organisation for the employed (all $\mathrm{p}>0.22$ ) and for homemakers, effective organisation was related only to social leisure (exercise and self-focused: $p>0.05$, social leisure: $p=0.001$ ).

Finally, as previously reported, ${ }^{21}$ recreational activities had significant negative associations with depressive symptoms (see table 2) for the employed across all types of recreational activities (all $\mathrm{p}<0.001$ ), while after Bonferroni correction

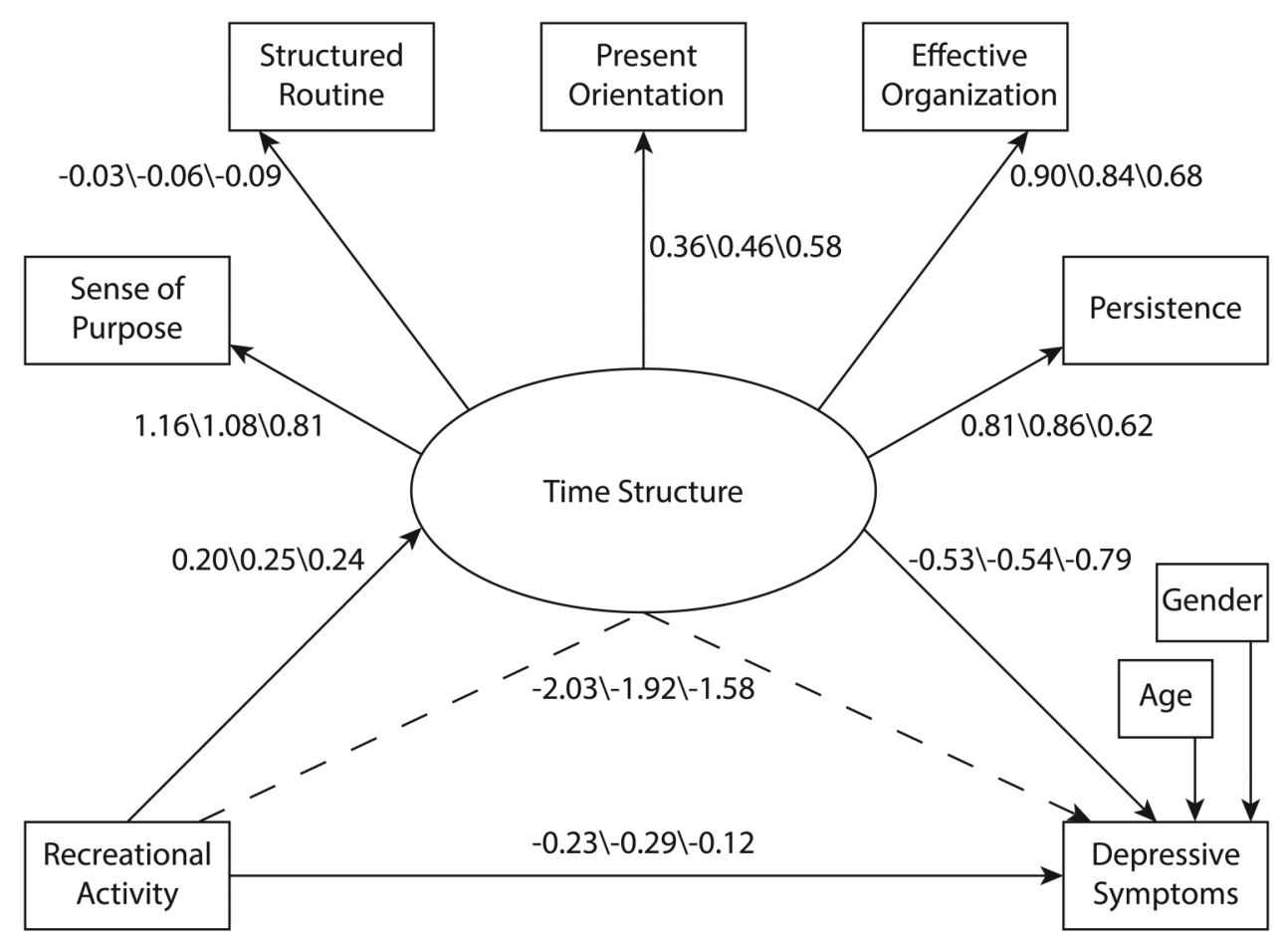

Figure 1 Models computed for employed only; presented are standardised coefficients for each of the three recreational activity types (Self-focused, Social and Exercise). The unstandardised indirect effects of recreational activity through time structure on depressive symptoms are represented by the dashed arrow. 


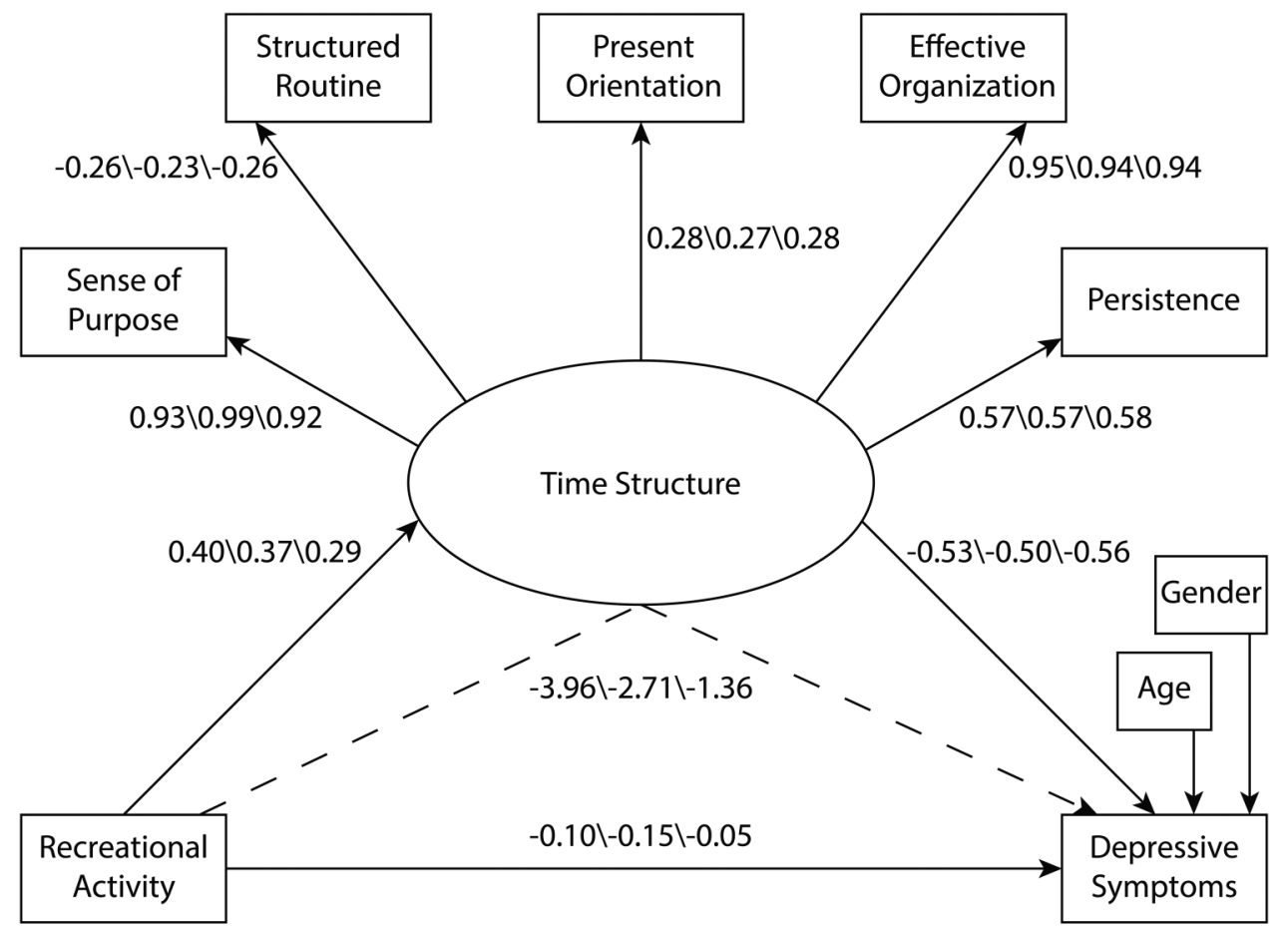

Figure 2 Models computed for unemployed only; presented are standardised coefficients for each of the three recreational activity types (Self-focused, Social and Exercise). The unstandardised indirect effects of recreational activity through time structure on depressive symptoms are represented by the dashed arrow.

$(\alpha=0.003)$, exercise was no longer significantly associated with depressive symptoms for unemployed individuals (social and self-focused: $p<0.001$; exercise: $p=0.009$ ). Examining homemakers revealed a pattern similar to the employed group (all $\mathrm{p}<0.002)$. Covariance matrices for each group are presented in online supplementary table S1.

\section{Hypotheses testing}

Mediation

To determine how the relationships between leisure (social, selffocused and exercise) activities and depressive symptoms are influenced by time structure, and whether these relationships differ for employed and unemployed individuals, we examined the effect of

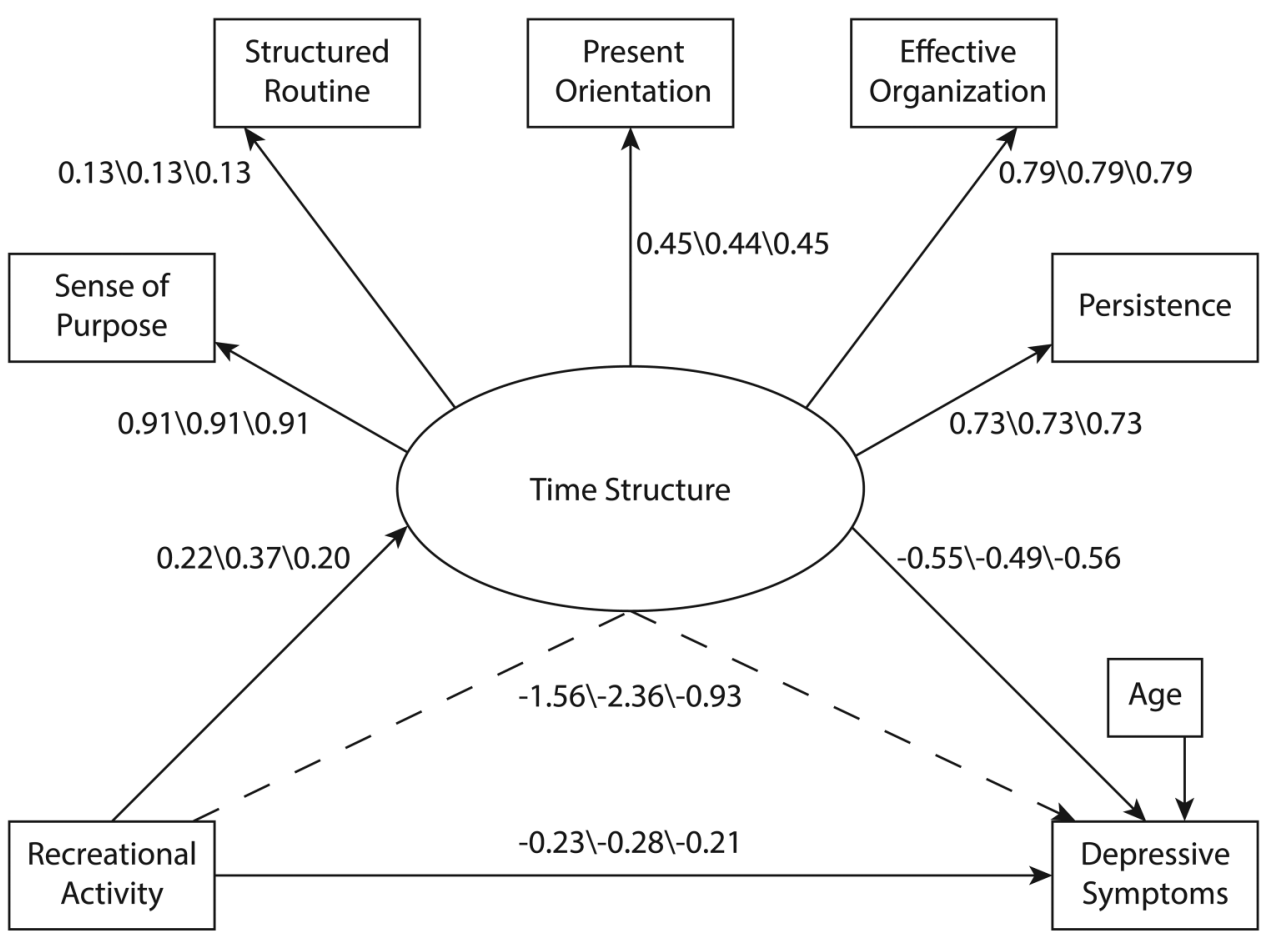

Figure 3 Models computed for homemakers only; presented are standardised coefficients for each of the three recreational activity types (Self-focused, Social and Exercise). The unstandardised indirect effects of recreational activity through time structure on depressive symptoms are represented by the dashed arrow. 
Table 2 Partial correlations controlling for gender and age

\begin{tabular}{|c|c|c|c|c|c|c|}
\hline & Sense of purpose & Structured routine & Present orientation & Effective organisation & Persistence & CESD \\
\hline \multicolumn{7}{|l|}{ Employed } \\
\hline Social & $0.348^{*}$ & -0.011 & 0.22 & 0.195 & 0.165 & $-0.454^{*}$ \\
\hline Self-focused & $0.284^{*}$ & -0.085 & 0.081 & 0.152 & 0.093 & $-0.354^{*}$ \\
\hline Exercise & $0.307^{*}$ & 0.056 & 0.128 & 0.135 & 0.124 & $-0.361^{*}$ \\
\hline CESD & $-0.705^{*}$ & 0.032 & $-0.483^{*}$ & $-0.513^{*}$ & $-0.457^{*}$ & \\
\hline \multicolumn{7}{|l|}{ Unemployed } \\
\hline Social & $0.398^{*}$ & -0.139 & 0.133 & $0.304^{*}$ & 0.126 & $-0.331^{*}$ \\
\hline Self-focused & $0.356^{*}$ & -0.154 & 0.139 & $0.414^{*}$ & 0.196 & $-0.312^{*}$ \\
\hline Exercise & 0.208 & -0.066 & -0.02 & $0.328^{*}$ & $0.217^{*}$ & -0.210 \\
\hline CESD & $-0.558^{*}$ & 0.223 & -0.187 & $-0.508^{*}$ & $-0.316^{*}$ & \\
\hline \multicolumn{7}{|l|}{ Homemaker } \\
\hline Social & $0.349^{*}$ & 0.023 & 0.077 & $0.316^{*}$ & 0.221 & $-0.464^{*}$ \\
\hline Self-focused & 0.163 & 0.124 & -0.031 & 0.186 & 0.105 & $-0.331^{*}$ \\
\hline Exercise & 0.156 & 0.030 & 0.023 & 0.089 & 0.127 & $-0.294^{*}$ \\
\hline CESD & $-0.545^{*}$ & -0.186 & -0.185 & $-0.457^{*}$ & $-0.416^{*}$ & \\
\hline
\end{tabular}

*Significant at a Bonferroni corrected level of 0.003 ; homemaker not controlled for gender.

CESD, Center for Epidemiologic Studies Depression.

introducing the latent variable of time structure. Models were examined for both direct (leisure activity $\rightarrow$ depressive symptoms) and indirect (leisure activity $\rightarrow$ time structure $\rightarrow$ depressive symptoms) effects on depressive symptoms, and statistical details are presented in online supplementary table S2 and figures 1-3.

Structural equation modelling analyses revealed that all models for the employed participants had generally poor fit (RMSEA $>0.12$ ), while all models for the unemployed participants had good to excellent model fit (RMSEA $<0.05$ ). For employed participants, we found evidence that time structure partially mediated the relationships between exercise, social and selffocused leisure activities and depressive symptoms. For unemployed participants, the relationship observed between exercise or self-focused leisure activity and depressive symptoms was completely mediated by the addition of time structure. Additionally, time structure partially mediated the relationship between social recreational activities and depressive symptoms. Assessing homemakers revealed that similar to employed participants, the relationships between all three leisure activities and depressive symptoms were partially mediated by the latent variable time structure. However, the model fits for all three activities were decent to good (RMSEA $<0.07$ ). Of note, the partial mediation effect of exercise was at the trend-level significant $(p=0.059)$, most likely attributable to the just barely significant direct contributions of exercise on time structure $(\beta=0.20, p=0.05)$.

Since the data were not obtained longitudinally, we also examined whether depressive symptoms were influencing leisure activities through changes in time structure. Only for unemployed participants, time structure partially mediated the association of depressive symptoms on social recreation $(\beta=-0.11, p=0.30)$, selffocused leisure $(\beta=-0.03, p=0.76)$ and exercise $(\beta=-0.00$, $\mathrm{p}=0.96$ ), with significant indirect effects (self $\mathrm{B}=-0.02, \mathrm{p}=0.001$; social: $\mathrm{B}=-0.02, \mathrm{p}=0.01$; exercise $\mathrm{B}=-0.03, \mathrm{p}=0.01)$. The lack of association between time structure and leisure activities in general $(\beta<0.15, p>0.18)$ was interpreted as indicative of the mostly unidirectional influence of recreational activities increasing time structure and not time structure increasing activity participation.

\section{Time structure}

The second aim of the current study was to examine the five subscales of the TSQ (sense of purpose, structured routine, present orientation, effective organisation and persistence) for their individual contributions towards the latent concept of time structure (see figures 1-3). Sense of purpose was used across all models involving TSQ as the primary indicator variable for the latent construct. For employed participants (figure 1), structured routine did not contribute significantly to any model (all $\mathrm{p}>0.33$ ). Interestingly, for unemployed participants (figure 2), structured routine contributed significantly to time structure for all three models examining self-focused leisure $(\beta=-0.26$, $\mathrm{p}=0.005)$, exercise $(\beta=-0.26, \mathrm{p}=0.005)$ and social recreation $(\beta=-0.23, p=0.009)$. No other factor differences were noted between employment groups for present orientation (all $\beta>0.27$ ), effective organisation (all $\beta>0.68$ ), or persistence (all $\beta>0.57$ ), and all were found to have a significant role in the formation of time structure. When examining the factors of time structure that composed the TSQ in homemakers, we again found a pattern similar to the employed group, that is, the structured routine did not significantly contribute to the latent variable time structure, while all other factors did (see figure 3).

\section{DISCUSSION}

This study used three populations known to report varying levels of time structure to evaluate whether leisure activities impose their mental health benefits through increasing individuals' perception of spending their time effectively. Unemployed participants reported significantly less time structure and more depressive symptoms than either the employed or homemakers. No differences were observed between the three employment groups for the frequency in which they engaged in leisure activities. In line with our hypothesis, but for the unemployed only, the mental health benefits of leisure activities were fully explained by higher engagement in all five facets of time structure. On the contrary, for the employed and homemakers, only four facets of time structure were relevant, and only partially explained the link between leisure activities and depressive symptoms.

\section{Meditational role of time structure}

Consistent with recent meta-analytical findings in depressed as well as non-clinical adult populations, ${ }^{37}{ }^{38}$ recreational activities had direct beneficial relationships with depressive symptoms in 
employed participants, ${ }^{20}$ while only some of these effects were exerted by increasing the time structure. ${ }^{39}$ As suggested previously, employment naturally provides time structure. ${ }^{10}$ Therefore, the importance of generating time structure through recreational activities may be reduced and, consequently, its protective role in the context of depressive symptoms. In contrast, for unemployed participants, mental health benefits of recreational activities were an indirect effect of recreational activities providing time structure. This was true for self-focused leisure activities and exercise, that is, participating in self-focused leisure activities or exercise directly benefited unemployed individuals in terms of depressive symptoms. Good model fits in the unemployed group, and relatively poor model fits in employed participants further emphasised the strong difference in the health-relevant role of time structure.

These findings also support earlier interpretations that meaning assigned particularly to self-focused recreational activities may change due to employment status. ${ }^{21}$ For example, while watching TV may be a restorative activity after a long day at work, for someone who is unemployed, watching TV may lack such a purpose. The current findings confirm that if selffocused leisure activities do not serve the purpose of providing time structure, they also show no beneficial associations with depressive symptoms. Interestingly, while physical activity may generally be a successful intervention for depression, ${ }^{16} 1740$ exercise had no direct relationship with depressive symptoms for unemployed individuals. Instead, the imposition of structured routines, sense of purpose, or persistence induced by exercise programmes appeared to be the 'active ingredient' in terms of decreasing depressive symptoms. If confirmed in future studies, this observation has important implications for how to tailor exercise intervention programmes to make them most successful for various employment groups. Last, independent of employment status, social leisure activities showed both direct effects on depressive symptoms and indirect effects through increasing time structure. While the direct link between social leisure activities and depressive symptoms is in line with previous findings describing health benefits of social interactions, ${ }^{41}$ the current findings suggest that one of the mechanisms by which social leisure may buffer depressive symptoms is through the actions needed to participate in social leisure (eg, scheduling a dinner with friends). As such, they extend the current literature by suggesting a symbiotic nature of these two methods of action-direct and through time structure-in the context of depressive symptoms. As such, our results also suggest social leisure activities as a way to decrease the motivation-behaviour gap ${ }^{42}$ by providing an alternative approach to exercise as the currently promoted but higher-barrier mental health intervention.

Despite describing the employment status of over 12 million people in 2012, ${ }^{43}$ homemakers represent an understudied group. In the present study, the mediational patterns for homemakers resembled patterns found in employed participants. This circumstance may largely be due to the fact that the majority of the assessed homemakers (84\%) reported children living in their household. Homemakers with children likely have regularly structured days, and thus, similar experiences as employed individuals in terms of time structure. However, the model fits for homemakers more closely approximated those observed in the unemployed, suggesting that the source of time structure may be an important consideration. While employed individuals are likely to have time structure imposed externally from their jobs, homemakers must generate their own internal sense of time structure. In future studies it would thus be interesting to further examine how internally versus externally generated time structure may influence the relationships with health. Furthermore, while meditational patterns in homemakers resembled those observed in employed individuals when assessing self-focused and social activities, the patterns differed for exercise. Specifically, in homemakers, exercise was significantly related to depressive symptoms, but effects through time structure were only marginal. This suggests an alternative mechanism, such as physical fitness or stress reduction, underlying the beneficial link between exercise and depressive symptoms in homemakers.

\section{Time structure facets}

A distinct pattern emerged across employment groups regarding which facets contributed to the overall construct of time structure. As previously described, increased sense of purpose, ${ }^{24}$ effective organisation, ${ }^{23}$ and persistence ${ }^{26}$ were all negatively associated with depressive symptoms independent of employment status. However, the current study confirms all three facets as main contributors to the latent variable of time structure across the three employment groups. In contrast, present orientation made only relatively moderate contributions, while differences between employment groups emerged in how the facet of structured routine contributed to the overall construct of time structure. Specifically, while unrelated in the employed and homemakers, structured routine had a small but significant contribution to time structure in the unemployed. This suggests that daily routines provided by being employed, or a homemaker involved in childcare, may not per se be health protective. However, once lost, such as the result of becoming unemployed, daily routines become a health-relevant factor.

\section{Limitations}

In addition to the limitations due to the cross-sectional nature of the study, the findings of the present study are based on correlational data, and although poorly fitting alternative models suggest that participation in recreational activities is helping to ameliorate depressive symptoms, bidirectional relationships established in previous literature cannot be discounted. ${ }^{39}$ Furthermore, all participants reported relatively high levels of depressive symptoms. While a potential theoretical concern, these scores also emphasise the clinical relevance of our findings. Finally, while women represent the majority of 'stay at home' parents, ${ }^{43}$ and therefore our findings are applicable to the majority of homemakers, future studies may expand this line of research to childless and male homemakers.

\section{CONCLUSION}

Current research has focused on recreational activities, specifically exercise, ${ }^{16}$ for prospective depression ameliorating effects. The current study extended those findings to two understudied groups, unemployed individuals and homemakers, and differentiated between facets of recreational activities. Most importantly, however, it examined the question whether beneficial effects of recreational activities may have been caused by contributions to time structure.

The present findings suggest that independent of employment status, a stronger sense of purposefully using one's time is linked to lower depressive symptoms. For employed individuals, our results further support previous reports of recreational activities showing beneficial associations with depressive symptoms. This was true for above and beyond the effects of time structure. The current study extended these observations to homemakers. For unemployed individuals, however, a different 
pattern emerged. Here, time structure was the central beneficial factor in terms of depressive symptoms; recreational activities were only beneficial to the extent that they contributed to a stronger sense of time structure.

In terms of applications, promoting recreational activities may not only benefit mental health in employed individuals, but in homemakers as well. Furthermore, given the central role of time structure in depressive symptoms risk for the unemployed, promoting social activities that provide a sense of daily structure may be a promising low-cost intervention to improve mental health in this population.

\section{What is already known on this subject}

Exercise and leisure activities (social and self-focused) are receiving increasing attention as alternative non-pharmacological approaches to treating depression. Among possible mechanisms underlying this relationship, indirect evidence suggests that the time structure provided by recreational activities may be an important factor leading to mental health benefits. Interestingly, participation in recreational activities, sense of time structure, and depressive symptom risk all vary by employment status. Hence, the current study aimed at testing whether fewer leisure activities may contribute to higher risk for negative mental health by decreased time structure, and whether these associations differ between the employed, unemployed and homemakers. To address these questions, we used Amazon Mechanical Turk to assess depressive symptoms, leisure activities and time structure in 406 participants (155 unemployed, 140 employed and 111 homemakers).

\section{What this study adds}

For the employed and homemakers, time structure only partially explained the link between leisure activities and depressive symptoms, suggesting that time structure is only one mechanism among others. On the contrary, for unemployed individuals, mental health benefits of recreational activitiesincluding exercise and social leisure-appear to be secondary to those activities providing a strong sense of time structure. If confirmed in longitudinal studies, our findings suggest that promoting recreational activities may not only benefit mental health in employed individuals, but in homemakers as well. For the unemployed, promoting social activities that provide a sense of daily structure may be a more promising approach to improving mental health.

Funding This work was supported by the National Institute of General Medical Sciences (NIGMS) 'Brain-Body-Behavior Interface in Learning and Development Across the Lifespan' training grant T32GM084907 (AMG).

Competing interests None declared.

Ethics approval Brandeis University Institutional Review Board.

Provenance and peer review Not commissioned; externally peer reviewed.

\section{REFERENCES}

1 Gabrys L, Michallik L, Thiel C, et al. Effects of a structured physical-activity counseling and referral scheme in long-term unemployed individuals: A pilot accelerometer study. Behav Med 2013;39:44-50
2 Howe GW, Hornberger AP, Weihs K, et al. Higher-order structure in the trajectories of depression and anxiety following sudden involuntary unemployment. J Abnorm Psychol 2012;121:325-38.

3 Marcotte DE, Wilcox-Gök V, Redmon DP. Prevalence and patterns of major depressive disorder in the United States labor force. J Ment Health Policy Econ 1999:2:123-31.

4 McKee-Ryan F, Song Z, Wanberg CR, et al. Psychological and physical well-being during unemployment: a meta-analytic study. J App/ Psychol 2005;90:53.

5 Song MR, Lee YS, Baek JD, et al. Physical activity status in adults with depression in The National Health and Nutrition Examination Survey, 2005-2006. Public Health Nurs 2012;29:208-17.

6 Waters L, Moore K. Self-esteem, appraisal and coping: a comparison of unemployed and re-employed people. J Organ Behav 2002;23:593-604.

7 Hammer T. Unemployment and mental health among young people: a longitudinal study. J Adolesc 1993;16:407-20.

8 Paul Kl, Batinic B. The need for work: Jahoda's latent functions of employment in a representative sample of the German population. J Organ Behav 2010;31:45-64.

9 Wanberg CR, Griffiths RF, Gavin MB. Time structure and unemployment: A longitudinal investigation. Journal of Occupational and Organizational Psychology 1997;70:75-95.

10 Paul Kl, Geithner E, Moser K. Latent deprivation among people who are employed, unemployed, or out of the labor force. J Psychol Interdiscip Appl 2009;143:477-91.

11 Creed PA, Macintyre SR. The relative effects of deprivation of the latent and manifest benefits of employment on the well-being of unemployed people. J Occup Health Psychol 2001;6:324-31.

12 Anderson RJ, Brice $S$. The mood-enhancing benefits of exercise: memory biases augment the effect. Psychol Sport Exerc 2011;12:79-82.

13 Hogan CL, Mata J, Carstensen LL. Exercise holds immediate benefits for affect and cognition in younger and older adults. Psychol Aging 2013;28:587-94.

14 Ruby MB, Dunn EW, Perrino A, et al. The invisible benefits of exercise. Health Psychol 2011;30:67-74.

15 Blumenthal JA, Babyak MA, Doraiswamy PM, et al. Exercise and pharmacotherapy in the treatment of major depressive disorder. Psychosom Med 2007;69:587-96.

16 Craft LL, Perna FM. The benefits of exercise for the clinically depressed. Prim Care Companion J Clin Psychiatry 2004;6:104-11.

17 Martinsen EW. Physical activity in the prevention and treatment of anxiety and depression. Nord J Psychiatry 2008;62(Suppl 47):25-9.

18 Babyak M, Blumenthal JA, Herman S, et al. Exercise treatment for major depression: maintenance of therapeutic benefit at 10 months. Psychosom Med 2000;62:633-8.

19 Ehrenreich-May J, Bilek EL. Universal prevention of anxiety and depression in a recreational camp setting: An initial open trial. Child Youth Care Forum 2011;40:435-55.

20 Patten SB, Williams JVA, Lavorato DH, et al. Recreational physical activity ameliorates some of the negative impact of major depression on health-related quality of life. Front Psychiatry 2013;4:22.

21 Goodman WK, Geiger AM, Wolf JM. Differential links between leisure activities and depressive symptoms in unemployed individuals. J Clin Psychol 2016;72:70-8.

22 Selenko E, Batinic B, Paul K. Does latent deprivation lead to psychological distress? Investigating Jahoda's model in a four-wave study. J Occup Organ Psychol 2011;84:723-40

23 Bond MJ, Feather NT. Some correlates of structure and purpose in the use of time. J Pers Soc Psychol 1988;55:321-9.

24 Boyle PA, Barnes LL, Buchman AS, et al. Purpose in life is associated with mortality among community-dwelling older persons. Psychosom Med 2009;71:574-9.

25 Kelly WE. No time to worry: The relationship between worry, time structure, and time management. Pers Individ Dif 2003;35:1119-26.

26 Van Hoye G, Lootens H. Coping with unemployment: Personality, role demands, and time structure. J Vocat Behav 2013;82:85-95.

27 Chang A, Nguyen LT. The mediating effects of time structure on the relationships between time management behaviour, job satisfaction, and psychological well-being. Aust J Psychol 2011;63:187-97.

28 Vodanovich SJ, Seib HM. Relationship between time structure and procrastination. Psychol Rep 1997;80:211-15.

29 Mudrack PE. The structure of perceptions of time. Educ Psychol Meas 1997:57:222-40

30 Pressman SD, Matthews KA, Cohen S, et al. Association of enjoyable leisure activities with psychological and physical well-being. Psychosom Med 2009;71:725.

31 Hutchinson SL, Loy DP, Kleiber DA, et al. Leisure as a coping resource: variations in coping with traumatic injury and illness. Leisure Sci 2003;25:143.

32 Evans 0, Steptoe A. The contribution of gender-role orientation, work factors and home stressors to psychological well-being and sickness absence in male- and female-dominated occupational groups. Soc Sci Med 2002;54:481-92.

33 Buhrmester M, Kwang T, Gosling SD. Amazon's Mechanical Turk a new source of inexpensive, yet high-quality, data? Perspect Psychol Sci 2011;6:3-5.

34 Ross J, Zaldivar A, Irani L, et al. Who are the turkers? Worker demographics in Amazon Mechanical Turk. Irvine, USA: Department of Informatics, University of California; Tech. Rep, 2009

35 Radloff LS. The CES-D scale a self-report depression scale for research in the general population. Appl Psychol Meas 1977;1:385-401. 
36 Kenny D. Measuring model fit. Secondary Measuring model fit. 24 November 2015 http://davidakenny.net/cm/fit.htm

37 Schuch FB, Vancampfort D, Richards J, et al. Exercise as a treatment for depression: A meta-analysis adjusting for publication bias. J Psychiatr Res 2016;77:42-51.

38 Rebar AL, Stanton R, Geard D, et al. A meta-meta-analysis of the effect of physical activity on depression and anxiety in non-clinical adult populations. Health Psychol Rev 2015;9:366-78.

39 Waters L, Moore K. Reducing latent deprivation during unemployment: the role of meaningful leisure activity. J Occup Organ Psychol 2002;75:15-32.
40 Dunn AL, Trivedi MH, Kampert JB, et al. Exercise treatment for depression: efficacy and dose response. Am J Prev Med 2005;28:1-8.

41 Coleman D, Iso-Ahola SE. Leisure and health: the role of social support and self-determination. J Leisure Res 1993:25:111-28.

42 Brug J, Oenema A, Ferreira I. Theory, evidence and Intervention mapping to improve behavior nutrition and physical activity interventions. Int I Behav Nutr Phys Act 2005;2:2.

43 Galley J. Stay-at-home mothers through the years. U.S. Bureau of Labor Statistics, 2014. 